\title{
FILMES DE METAL-HEXACIANOFERRATO: UMA FERRAMENTA EM QUÍMICA ANALÍTICA
}

\author{
Ivanildo Luiz de Mattos* e Lo Gorton \\ Department of Analytical Chemistry, Lund University, Box 124, SE-221 00, Lund - Sweden
}

Recebido em 26/11/99; aceito em 11/12/00

\begin{abstract}
METAL-HEXACYANOFERRATE FILMS: A TOOL IN ANALYTICAL CHEMISTRY. Chemically modified electrodes based on hexacyanometalate films are presented as a tool in analytical chemistry. Use of amperometric sensors and/or biosensors based on the metal-hexacyanoferrate films is a tendency. This article reviews some applications of these films for analytical determination of both inorganic (e.g. $\mathrm{As}^{3+}$, $\mathrm{S}_{2} \mathrm{O}_{3}{ }^{2-}$ ) and organic (e.g. cysteine, hydrazine, ascorbic acid, gluthatione, glucose, etc.) compounds.
\end{abstract}

Keywords: metal hexacyanoferrate; modified electrodes; biosensor.

\section{INTRODUÇÃO}

Apesar de a história da química de hexacianoferratos (HCF) datar de 1704 com o descobrimento do azul da Prússia ("Prussian Blue", PB) ${ }^{1}$ somente recentemente tem havido um maior conhecimento das características eletroquímicas dos HCF's, especialmente daqueles em que os filmes são "insolúveis" em solução ${ }^{1}$. Desde o primeiro trabalho versando sobre o PB modificando superfícies de eletrodos ${ }^{2}$ tem havido um maior interesse na preparação eletroquímica, bem como, propriedades eletrocrômicas, eletrocatalítica ou caracterização espectroscópica do PB e seus análogos ${ }^{3-14}$.

Férrico-ferrocianeto ${ }^{12,15-18}$ e seus análogos (substituídos com $\mathrm{Cu}^{19}, \mathrm{Ni}^{20}, \mathrm{Co}^{21}, \mathrm{Ru}+\mathrm{In}^{22}, \mathrm{Mo}^{23}, \mathrm{~V}^{24}$, etc.) tem contribuído para incrementar o número de trabalhos versando sobre a modificação eletroquímica das superfícies dos mais variados materiais de eletrodo. Este interesse tem aumentado na medida em que se obtém filmes com características eletroquímicas muito bem definidas tanto a nível dos processos de oxidação-redução e propriedades de troca-iônica quanto da intercalação de componentes ${ }^{19,25}$

De maneira geral, pode-se apresentar a fórmula de um filme de hexacianometalato como:

$$
\left(\mathrm{M}_{1}{ }^{\mathrm{A}}\right)_{\mathrm{k}}\left[\mathrm{M}_{2}{ }^{\mathrm{B}}(\mathrm{CN})_{\mathrm{m}}\right]_{\mathrm{p}} \cdot \mathrm{xH}_{2} \mathrm{O}
$$

onde $M_{1}$ e $M_{2}$ referem-se aos metais (idênticos ou diferentes) com valências $\mathrm{A}$ e $\mathrm{B} ; \mathrm{k}, \mathrm{m}$, e p indicam a estequiometria do complexo, os quais são formados pela mistura de soluções de $\mathrm{M}_{2}{ }^{\mathrm{B}}(\mathrm{CN})_{6}{ }^{\mathrm{n}-}$ com um sal apropriado de $\mathrm{M}_{1}{ }^{\mathrm{A}}$. Se $\mathrm{M}_{1}$ e $\mathrm{M}_{2}$ são metais de transição, existe a formação de inúmeros componentes com variações tanto dos íons metálicos quanto de suas valências ${ }^{2,9,26}$

Dentre os sensores baseados nestes filmes de HCF, destacam-se aqueles modificados com filmes de $\mathrm{Fe}^{\mathrm{III}}{ }_{4}\left[\mathrm{Fe}^{\mathrm{II}}(\mathrm{CN})_{6}\right]_{3}$ para catalisar a redução ou oxidação de dióxido de carbono ${ }^{27,28}$ hidrazina $^{29}$, oxigênio molecular ${ }^{30}$, peróxido de hidrogênio ${ }^{31,32}$ etc. Entre as inúmeras aplicações, a quantificação de $\mathrm{H}_{2} \mathrm{O}_{2}$ tem se destacado pois sua determinação além de ser essencial nos estudos bio-médicos e do meio-ambiente ${ }^{33}$, tem demonstrado importância no processamento industrial de alimentos ${ }^{34}$, medicamentos $^{35}$, etc. Somado a isto, peróxido de hidrogênio está presente em inúmeras reações biológicas como produto de várias oxidases, sendo portanto um importante parâmetro para suas quantificações ${ }^{36,37}$.

\footnotetext{
* Endereço Atual: Departamento de Química, Faculdade de Ciências Universidade Estadual Paulista, CP 473, 17033-360 Bauru - SP, Brasi
}

Com estes fatos em mente, o objetivo deste texto foi o de apresentar uma breve revisão destes filmes de hexacianoferrato, demonstrando suas potencialidades como ferramenta para diversas aplicações em química analítica. Em função do elevado número de trabalhos versando sobre o tema, foram selecionadas algumas das mais recentes aplicações focalizando o aspecto analítico. Assim, foram apresentadas aplicações dos filmes de hexacianoferrato baseado nos metais $\mathrm{Fe}, \mathrm{Cu}, \mathrm{Ni}, \mathrm{Co}, \mathrm{Mn}, \mathrm{Cr}$, $\mathrm{Zn}, \mathrm{Ag}, \mathrm{Cd}$ e In em determinações amperométricas, potenciométricas e espectrofotométricas tanto de componentes orgânicos quanto de inorgânicos.

Foram incluídas algumas considerações práticas na obtenção destes filmes de HCF objetivando demonstrar, entre outras, suas características eletroquímicas. Considerando-se que os filmes baseados em $\mathrm{Fe}$ (III) têm sido os mais investigados dentre todos os hexacianometalatos, suas características eletroquímicas foram apresentadas de forma um pouco mais extensa em relação aos demais.

\section{ALGUMAS CARACTERÍSTICAS ELETROQUÍMICAS DOS FILMES}

\section{1. $\mathrm{Fe}(\mathrm{III}) / \mathrm{Fe}(\mathrm{CN})_{6}$}

Quando um eletrodo de carbono vítreo é tratado a potencial da ordem de $+0,4 \mathrm{~V}$ vs. ECS em solução aquosa (meio ácido, pois em meio alcalino há a destruição do filme: $\mathrm{Fe}(\mathrm{CN})_{6}{ }^{4-}+$ $2 \mathrm{OH}^{-} \rightarrow \mathrm{Fe}(\mathrm{OH})_{2}+6 \mathrm{CN}^{-} ; 2 \mathrm{Fe}(\mathrm{OH})_{2}+\mathrm{H}_{2} \mathrm{O}+1 / 2 \mathrm{O}_{2} \rightarrow$ $\left.2 \mathrm{Fe}(\mathrm{OH})_{3}\right)$ contendo íons $\mathrm{Fe}^{3+}$ e $\left[\mathrm{Fe}(\mathrm{CN})_{6}\right]^{3-}$, observa-se a formação do filme de FeHCF na superfície do eletrodo.

Considerando-se que a espécie eletrodepositada é férricoferrocianeto ou seja, $\mathrm{Fe}^{\mathrm{III}}{ }_{4}\left[\mathrm{Fe}^{\mathrm{II}}(\mathrm{CN})_{6}\right]_{3}$, o valor de potencial empregado tem por objetivo reduzir os íons $\mathrm{Fe}(\mathrm{CN})_{6}{ }^{3-}$ em $\mathrm{Fe}(\mathrm{CN})_{6}^{4-}$ desde que o equilíbrio $\left[\mathrm{Fe}(\mathrm{CN})_{6}\right]^{3-}+\mathrm{e}^{-} \leftrightarrow\left[\mathrm{Fe}(\mathrm{CN})_{6}\right]^{4-}$ apresenta $\mathrm{E}^{\circ}=+0,36 \mathrm{~V}$.

Desta forma, na presença de $\left[\mathrm{Fe}(\mathrm{CN})_{6}\right]^{4-}$ (reduzido eletroquimicamente) e íons $\mathrm{Fe}^{3+}$, há a formação do filme de azul da Prússia na superfície do eletrodo. A estabilidade operacional deste filme ${ }^{46}$ vai depender, entre outros fatores, do procedimento de deposição (potenciostática ou galvanostática, etapas que envolvem fixação do potencial ou corrente respectivamente), da natureza dos reagentes, do procedimento de armazenamento do filme formado, etc. Caso este eletrodo modificado seja submetido à varredura de potencial entre $-0,05$ e $+0,35 \mathrm{~V}$ vs. ECS (em solução $0,1 \mathrm{M} \mathrm{KCl}$ ) é possível identificar dois picos $(c a .+0,15 \mathrm{e}+0,12 \mathrm{~V})$ associados à oxidação e redução, respectivamente ${ }^{32}$. Algumas características interessantes destes filmes podem ser relatadas como: 
i) $\mathrm{Fe}^{\mathrm{III}}{ }_{4}\left[\mathrm{Fe}^{\mathrm{II}}(\mathrm{CN})_{6}\right]_{3}$ (“insolúvel”) e $\mathrm{K}\left\{\mathrm{Fe}^{\mathrm{III}}\left[\mathrm{Fe}^{\mathrm{II}}(\mathrm{CN})_{6}\right]\right\}$ ("solúvel”) são as duas fórmulas apresentadas para o PB. O termo "solubilidade" refere-se à tendência do mesmo em formar solução coloidal ${ }^{38}$. Por apresentar menor tendência, o filme de $\mathrm{Fe}^{\mathrm{III}}{ }_{4}\left[\mathrm{Fe}^{\mathrm{iI}}(\mathrm{CN})_{6}\right]_{3}$ tem sido o mais freqüentemente empregado para a modificação das superfícies dos eletrodos ${ }^{32}$.

ii) o precipitado de $\mathrm{PB}$ contém em média 14 moléculas de água por unidade $\left(\mathrm{Fe}_{4}\left[\mathrm{Fe}(\mathrm{CN})_{6}\right]_{3} \cdot 14 \mathrm{H}_{2} \mathrm{O}\right)$ as quais, quando eliminadas, favorecem o incremento da eletroatividade e estabilidade operacional dos filmes ${ }^{4,32}$.

iii) o PB pode ser oxidado em verde da Prússia ("Prussian Green", PG) assim como pode ser reduzido à branco da Prússia ("Prussian White", PW ou sal de Everitt) a potenciais da ordem de $+0,7$ e $-0,1 \mathrm{~V}$ vs. ECS. As reações de oxidação e redução podem ser apresentadas como ${ }^{33}$ :

$$
\mathrm{Fe}^{\mathrm{III}}{ }_{4}\left[\mathrm{Fe}^{\mathrm{II}}(\mathrm{CN})_{6}\right]_{3}+4 \mathrm{~K}^{+}+4 \mathrm{e}^{-} \leftrightarrow \mathrm{K}_{4}\left\{\mathrm{Fe}_{4}^{\mathrm{II}}\left[\mathrm{Fe}^{\mathrm{II}}(\mathrm{CN})_{6}\right]_{3}\right\}
$$

O PW, apresenta atividade catalítica para a redução de oxigênio molecular e de peróxido de hidrogênio em meio ácido; atua como um catalisador para a redução de peróxido de hidrogênio presente em solução ou gerado durante o curso de uma reação enzimática ${ }^{36}$. Desde que este processo eletrocatalítico ocorre a potencial da ordem de $-0,1$ V vs. ECS, é possível eliminar a influência de espécies interferentes e desenvolver sensores com superfície catalítica para a determinação de $\mathrm{H}_{2} \mathrm{O}_{2}$ a baixo potencial ${ }^{40}$. Saliente-se que em certas condições de eletrodeposição, os filmes de $\mathrm{PB}$ podem catalisar de forma seletiva a eletro-redução de peróxido de hidrogênio em presença de oxigênio molecular ${ }^{32}$.

\section{2. $\mathrm{Cu}(\mathrm{II}) / \mathrm{Fe}(\mathrm{CN})_{6}$}

Se um eletrodo de carbono vítreo modificado com filme de $\mathrm{CuHCF}$ for inserido em solução aquosa $0,5 \mathrm{M} \mathrm{K}_{2} \mathrm{SO}_{4}$ e for submetido à varredura de potencial entre $-0,2$ e $+0,95 \mathrm{~V}$ vs. ECS, será observada a presença de picos com potencial da ordem de $+0,69 \mathrm{~V}$ vs. ECS $\left(\Delta \mathrm{E}_{\mathrm{p}}=0\right)$, correspondendo à oxidação e redução, respectivamente ${ }^{26}$ :

$$
\mathrm{Cu}_{3}{ }_{3}\left[\mathrm{Fe}^{\mathrm{III}}(\mathrm{CN})_{6}\right]_{2}+2 \mathrm{e}^{-}+2 \mathrm{~K}^{+} \leftrightarrow \mathrm{K}_{2}\left\{\mathrm{Cu}_{3}{ }_{3}\left[\mathrm{Fe}^{\mathrm{II}}(\mathrm{CN})_{6}\right]_{2}\right\}
$$

Saliente-se que neste potencial $(+0,69 \mathrm{~V})$, o processo de oxiredução envolve a mudança dos estados de oxidação de $\mathrm{Fe}^{\mathrm{III}} /$ $\mathrm{Fe}^{\mathrm{II}}$ do filme e não do par $\mathrm{Cu}^{\mathrm{II}} / \mathrm{Cu}^{\mathrm{I}}$. Nota-se também que durante o processo de redução, íons $\mathrm{K}^{+}$são incorporados ao filme e contribuem para manter sua neutralidade e estabilidade ${ }^{26,58}$.

\section{3. $\mathrm{Ni}(\mathrm{II}) / \mathrm{Fe}(\mathrm{CN})_{6}$}

Quando o filme de NiHCF (eletrodepositado em eletrodo de carbono) é submetido à varredura de potencial entre $0,0 \mathrm{e}+1,0$ $\mathrm{V}$ vs. ECS em solução $0,5 \mathrm{M} \mathrm{KCl}$, observam-se picos com potenciais da ordem de $+0,4$ e $+0,6 \mathrm{~V}$. O perfil destes picos é alterado com a velocidade de varredura e concentração do eletrólito ${ }^{7}$. De forma geral, a reação envolvida no eletrodo pode ser representada como:

$$
\mathrm{K}\left\{\mathrm{Ni}^{\mathrm{II}}{ }_{2}\left[\mathrm{Fe}^{\mathrm{III}}(\mathrm{CN})_{6}\right]\right\}+\mathrm{K}^{+}+\mathrm{e}^{-} \leftrightarrow \mathrm{K}_{2}\left\{\mathrm{Ni}^{\mathrm{II}}\left[\mathrm{Fe}^{\mathrm{II}}(\mathrm{CN})_{6}\right]\right\}
$$

\section{4. $\mathrm{Co}(\mathrm{II}) / \mathrm{Fe}(\mathrm{CN})_{6}$}

Após a eletropolimerização de CoHCF em carbono vítreo, pode ser identificado um par de picos correspondentes a $+0,6$ e $+0,3 \mathrm{~V}$ vs. $\mathrm{Ag} / \mathrm{AgCl}$, os quais estão associados à formação de $\mathrm{K}\left\{\mathrm{Co}^{\mathrm{II}}\left[\mathrm{Fe}^{\mathrm{III}}(\mathrm{CN})_{6}\right]\right\}$ e $\mathrm{K}_{2}\left\{\mathrm{Co}^{\mathrm{II}}\left[\mathrm{Fe}^{\mathrm{II}}(\mathrm{CN})_{6}\right]\right\}$ respectivamente. As equações referentes à deposição eletroquímica e do processo de oxidação/redução na superfície do eletrodo podem ser apresentadas como ${ }^{41}$ :

$$
\begin{aligned}
& \mathrm{Fe}^{\mathrm{II}}(\mathrm{CN})_{6}{ }^{4-}+\mathrm{Co}^{2+}+2 \mathrm{~K}^{+} \leftrightarrow \mathrm{K}_{2}\left\{\mathrm{Co}^{\mathrm{II}}\left[\mathrm{Fe}^{\mathrm{II}}(\mathrm{CN})_{6}\right]\right\} \\
& \mathrm{K}\left\{\mathrm{Co}^{\mathrm{II}}\left[\left(\mathrm{Fe}^{\mathrm{III}}(\mathrm{CN})_{6}\right]\right\}+\mathrm{K}^{+}+\mathrm{e}^{-} \leftrightarrow \mathrm{K}_{2}\left\{\mathrm{Co}^{\mathrm{II}}\left[\left(\mathrm{Fe}^{\mathrm{II}}(\mathrm{CN})_{6}\right]\right\}\right.\right.
\end{aligned}
$$

\section{5. $\mathrm{Cr}(\mathrm{III}) / \mathrm{Fe}(\mathrm{CN})_{6}$}

O filme de $\mathrm{CrHCF}, \mathrm{K}\left\{\mathrm{Cr}^{\mathrm{III}}\left[\mathrm{Fe}^{\mathrm{II}}(\mathrm{CN})_{6}\right]\right\}$ pode ser obtido em eletrodo de carbono vítreo varrendo-se o potencial entre $-0,2$ e $+0,95 \mathrm{~V}$ vs. ECS em solução aquosa contendo íons $\mathrm{Cr}^{3+} \mathrm{e}$ $\left[\mathrm{Fe}(\mathrm{CN})_{6}\right]^{3-}$ (meio ácido). Um voltamograma típico do filme em $0,1 \mathrm{M} \mathrm{KCl}$, indica que os dois picos oxidativos (para potencial aplicado mais positivo) do $\mathrm{Cr}^{\mathrm{II}}\left[\mathrm{Fe}^{\mathrm{II}}(\mathrm{CN})_{6}\right]$ estão a $+0,23$ e $+0,88 \mathrm{~V}$ enquanto que os dois picos redutivos aparecem a $+0,19$ e $+0,84$ V vs. ECS ${ }^{42}$. Na comparação dos voltamogramas do filme de $\mathrm{K}\left\{\mathrm{Cr}^{\mathrm{III}}\left[\mathrm{Fe}^{\mathrm{II}}(\mathrm{CN})_{6}\right]\right\}$ e do $\mathrm{Cr}\left(\mathrm{NO}_{3}\right)_{3}$ seriam observadas diferenças bastante significativas, já que o $\mathrm{Cr}\left(\mathrm{NO}_{3}\right)_{3}$ não apresenta picos de oxi-redução, pelo menos, para as condições apresentadas para a obtenção do filme em questão. Os picos atribuídos ao filme de $\mathrm{CrHCF}$, devem-se aos diferentes estágios de valência, e sua reação redox pode ser representada como:

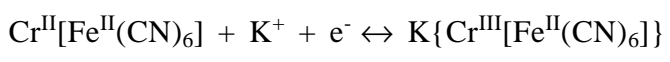

\section{6. $\operatorname{In}(\mathrm{III}) / \mathrm{Fe}(\mathrm{CN})_{6}$}

Quando um eletrodo de $\mathrm{SnO}_{2}$ é tratado a $+0,2 \mathrm{~V}$ vs. $\mathrm{Ag} /$ $\mathrm{AgCl}$ em solução aquosa (meio ácido) contendo íons $\mathrm{In}^{3+} \mathrm{e}$ $\left[\mathrm{Fe}(\mathrm{CN})_{6}\right]^{3-}$ observa-se a formação do filme de InHCF, $\mathrm{In}_{4}\left[\mathrm{Fe}(\mathrm{CN})_{6}\right]_{3}$. Inserindo-se este eletrodo modificado em solução $0,1 \mathrm{M} \mathrm{KCl}$ e variando-se o valor do potencial entre $+1,0$ e $+0,5 \mathrm{~V}$ vs. $\mathrm{Ag} / \mathrm{AgCl}$, observam-se picos separados em cerca de $0,1 \mathrm{~V}$. Nota-se que este grau de separação, entre outras características, é típico de filmes que apresentam elevada resistência ohmica $^{43,44}$.

A reação no eletrodo pode ser representada ${ }^{6}$ como:

$$
\mathrm{In}^{\mathrm{III}}\left[\mathrm{Fe}^{\mathrm{III}}(\mathrm{CN})_{6}\right]+\mathrm{K}^{+}+\mathrm{e}^{-} \leftrightarrow \mathrm{K}\left\{\operatorname{In}^{\mathrm{III}}\left[\mathrm{Fe}^{\mathrm{II}}(\mathrm{CN})_{6}\right]\right\}
$$

Saliente-se que as características eletroquímicas dos filmes variam com o procedimento de eletrodeposição empregado, com o tipo de eletrodo, eletrólito utilizado, etc. Por exemplo, nesta última consideração sobre os filmes de InHCF, se o mesmo for depositado em eletrodo de carbono vítreo, observa-se um pico a $+0,28 \mathrm{~V}$ vs. ECS referindo-se à redução do $\mathrm{HCF}(\mathrm{III})$; um pico a $+0,68 \mathrm{~V}$ correspondendo à oxidação do depósito inicial: $\mathrm{In}_{4}{ }^{\mathrm{III}}\left[\mathrm{Fe}^{\mathrm{II}}(\mathrm{CN})_{6}\right]_{3}$ - em presença de íons $\mathrm{K}^{+}$este depósito seria $\left\{\mathrm{K}\left[\mathrm{In}^{\mathrm{III}} \mathrm{Fe}^{\mathrm{II}}(\mathrm{CN})_{6}\right]\right\}$, e dois picos a $+0,75 \mathrm{~V}(\Delta \mathrm{Ep}=0)$ atribuídos ao par $\operatorname{In}^{\mathrm{III}}\left[\mathrm{Fe}^{\mathrm{III}}(\mathrm{CN})_{6}\right] / \mathrm{K}\left\{\operatorname{In}^{\mathrm{III}}\left[\mathrm{Fe}^{\mathrm{II}}(\mathrm{CN})_{6}\right]\right\}^{45}$.

\section{APLICAÇÕES}

Como primeira aplicação, pode ser citado o clássico trabalho de Itaya e colaboradores ${ }^{30}$ os quais demonstraram que a forma reduzida do $\mathrm{PB}$, apresentava atividade catalítica para a redução de oxigênio molecular e peróxido de hidrogênio em meio ácido, e que o processo de redução envolvia a transferência de quatro elétrons:

$$
\begin{gathered}
\mathrm{O}_{2}+4 \mathrm{H}^{+}+\mathrm{K}_{4}{ }^{+}\left\{\mathrm{Fe}^{\mathrm{II}}{ }_{4}\left[\mathrm{Fe}^{\mathrm{II}}(\mathrm{CN})_{6}\right]_{3} \cdot \mathrm{xH}_{2} \mathrm{O}\right\} \\
\mathrm{Fe}^{\mathrm{III}}{ }_{4}\left[\mathrm{Fe}^{\mathrm{II}}(\mathrm{CN})_{6}\right]_{3} \cdot \mathrm{xH}_{2} \mathrm{O}+2 \mathrm{H}_{2} \mathrm{O}+4 \mathrm{~K}^{+}
\end{gathered} \rightarrow
$$

Apesar de este trabalho não apresentar um enfoque analítico propriamente dito, ele vislumbrou as aplicações analíticas para a determinação de peróxido de hidrogênio nas mais variadas matrizes.

Neste contexto, como sensor para peróxido de hidrogênio, Lin e $\operatorname{Jan}^{21}$ apresentaram eletrodo de carbono vítreo modificado com filmes de CoHCF. O sensor apresentou tempo de resposta rápido $(6,5 \mathrm{~s})$, sensibilidade da ordem de $11,8 \mu \mathrm{Amm}^{-2} \mathrm{mM}^{-1} \mathrm{e}$ ausência de interferência dos ácidos ascórbico e úrico, assim 
como dopamina ao nível de $2,0 \times 10^{-4} \mathrm{M}$. Características analíticas comparáveis foram apresentadas pelo eletrodo de carbono vítreo modificado com filmes de $\mathrm{CrHCF}^{42}$. Neste caso o tempo de resposta e sensibilidade foram reportadas como 5,6 s e $11,52 \mu \mathrm{Amm}^{-2} \mathrm{mM}^{-1}$ respectivamente. Filmes de FeHCF também foram empregados para a determinação de peróxido de hidrogênio ${ }^{46}$ com sensibilidade inicial da ordem de $0,3 \mathrm{Acm}^{-2} \mathrm{M}^{-1}$.

Explorando a oxidação eletrocatalítica de hidrazina em eletrodo de carbono vítreo modificado com PB, Hou e Wang ${ }^{47}$ apresentaram sua determinação automatizada empregando os sistemas de análise por injeção em fluxo (FIA, do inglês, "flow-injection analysis"). Para incrementar a estabilidade do filme em regime em fluxo, depositaram sobre a superfície do eletrodo modificado membrana polimérica à base de Nafion ${ }^{\mathrm{MR}}$ (do inglês: "Nafion perfluorinated ion-exchange powder"). A estrutura do polímero pode ser apresentada como ${ }^{44}$ :

$$
\begin{aligned}
-\left(\mathrm{CF}_{2} \mathrm{CF}_{2) \mathrm{x}}\left(\mathrm{CF} \mathrm{CF}_{2}\right)_{\mathrm{y}}-\right. & \\
& \\
& \mathrm{O}-\left(\mathrm{C}_{3} \mathrm{~F}_{6}\right)-\mathrm{O}-\mathrm{CF}_{2} \mathrm{CF}_{2}-\mathrm{SO}_{3}{ }^{-}-
\end{aligned}
$$

e por apresentar-se carregada de carga negativa, membranas baseadas neste polímero tem sido cada vez mais usadas para incrementar a performance dos sensores e biossensores: após o depósito destas membranas sobre as enzimas imobilizadas na superfície dos eletrodos modificados, as cargas negativas das espécies interferentes - ácidos ascórbico e úrico, paracetamol, etc. - são prontamente repelidas ${ }^{46,56,58}$ resultando, entre outras características analíticas vantajosas, incremento da seletividade destes (bio)-sensores. Estudos realizados demonstraram que concentração de Nafion a $1 \%$ (v/v) eliminaram a interferência de ácido ascórbico, ácido úrico e paracetamol nas concentrações (doses terapêuticas no sangue) de $0,1 \mathrm{mM}, 0,5 \mathrm{mM}$ e $0,15 \mathrm{mM}$ respectivamente ${ }^{73}$.

Ainda investigando a oxidação de hidrazina, Narayanan e Scholtz ${ }^{20}$ empregaram eletrodos modificados com filmes de HCF em presença de diferentes metais, tais como $\mathrm{Cr}, \mathrm{Mn}, \mathrm{Fe}, \mathrm{Co}, \mathrm{Ni}$, $\mathrm{Cu}, \mathrm{Zn}, \mathrm{Ag}, \mathrm{Cd}$ e In. A atividade eletrocatalítica de cada filme, para oxidação do substrato, foi realizada sob potencial de pico e a potencial variado entre $+0,4 \mathrm{e}+0,7 \mathrm{~V}$ vs. ECS.

Fixando o potencial em $-0,1 \mathrm{~V}$ vs. $\mathrm{Ag} / \mathrm{AgCl}$, Lu e colaboradores apresentaram a determinação amperométrica automatizada de citocromo- $\mathrm{C}^{48}$ :

$$
\begin{aligned}
& \text { citocromo-C } \mathrm{C}_{\text {oxidada }}+\mathrm{K}_{4}\left\{\mathrm{Fe}^{\mathrm{II}}{ }_{4}\left[\mathrm{Fe}^{\mathrm{II}}(\mathrm{CN})_{6}\right]_{3}\right\} \rightarrow \\
& \mathrm{Fe}^{\mathrm{III}}{ }_{4}\left[\mathrm{Fe}^{\mathrm{II}}(\mathrm{CN})_{6}\right]_{3}+\text { citocromo- } \mathrm{C}_{\text {reduzida }}+4 \mathrm{~K}^{+}
\end{aligned}
$$

Espécies químicas, tais como $\mathrm{As}^{3+}, \mathrm{S}_{2} \mathrm{O}_{3}{ }^{2-}$, cisteína e 2furaldeído foram determinadas usando eletrodos de carbono vítreo modificados com filmes de InHCF combinado com $\mathrm{Ru}^{3+}$. A presença de rutênio foi relatada como responsável pelo incremento da estabilidade do filme em aplicações automatizadas ${ }^{22}$. A oxidação eletrocatalítica de $\mathrm{S}_{2} \mathrm{O}_{3}{ }^{2-}$ também foi realizada empregando-se eletrodos de carbono vítreo modificados com filmes de $\mathrm{HCF}^{49}$ em presença de Fe, In, Ni e Co. Filmes de NiHCF demonstraram potencialidade para a determinação de $\mathrm{S}_{2} \mathrm{O}_{3}{ }^{2-}$ em efluentes de águas industriais ${ }^{50}$ :

$$
\begin{gathered}
2 \mathrm{~K}\left\{\mathrm{Ni}^{\mathrm{II}}\left[\mathrm{Fe}^{\mathrm{III}}(\mathrm{CN})_{6}\right]\right\}+2 \mathrm{~S}_{2} \mathrm{O}_{3}{ }^{2-}+2 \mathrm{~K}^{+} \rightarrow \\
2 \mathrm{~K}_{2}\left\{\mathrm{Ni}^{\mathrm{II}}\left[\mathrm{Fe}^{\mathrm{II}}(\mathrm{CN})_{6}\right]\right\}+\mathrm{S}_{4} \mathrm{O}_{6}{ }^{2-}
\end{gathered}
$$

Cisteína, $n$-acetilcisteína e glutationa também foram determinadas em eletrodo de carbono vítreo modificado com $\mathrm{PB}^{51}$. Para incrementar estabilidade do filme em aplicações em fluxo à potenciais mais positivos do que $+0,9 \mathrm{~V}$ vs. $\mathrm{Ag} / \mathrm{AgCl}$, os eletrodos modificados foram recobertos com membrana à base de Nafion $^{\mathrm{MR}}$. Após separação cromatográfica e fixação do potencial em $+1,0 \mathrm{~V}$ vs. $\mathrm{Ag} / \mathrm{AgCl}$, foram incrementados os limites de detecção da ordem de pmol (V. tabela 1). Ainda explorando a hifenação de técnicas, tio-componentes também foram determinados empregando-se eletrodos de carbono vítreo modificados com filmes de $\mathrm{Cu}^{52}$ e $\mathrm{In}^{45} \mathrm{HCF}$ com aplicações em drogas e urinas. As equações química e de eletrodos podem ser apresentadas como:

$$
\begin{gathered}
\mathrm{In}^{\mathrm{III}} \mathrm{Fe}^{\mathrm{III}}(\mathrm{CN})_{6}+\underset{1 / 2}{\mathrm{R}-\mathrm{SH}}+\mathrm{K}^{+} \leftrightarrow \mathrm{K}_{1}\left\{\mathrm{In}^{\mathrm{III}}\left[\mathrm{Fe}^{\mathrm{II}}(\mathrm{CN})_{6}\right]\right\}+ \\
\mathrm{K}\left\{\mathrm{In}^{\mathrm{III}}\left[\mathrm{Fe}^{\mathrm{II}}(\mathrm{CN})_{6}\right]\right\} \leftrightarrow \mathrm{In}^{+}{ }^{\mathrm{III}} \mathrm{Fe}^{\mathrm{III}}(\mathrm{CN})_{6}+\mathrm{K}^{+}+\mathrm{e}^{-}
\end{gathered}
$$

onde, R-SH representa os quatro diferentes tio-componentes determinados (cisteína, glutationa, 6-tiopurina e metimazola). Tanto nesta aplicação, quando do uso de eletrodo modificado com filmes de $\mathrm{CuHCF}$, a presença de membrana polimérica (Nafion ${ }^{\mathrm{MR}}$ ) sobre a superfície do eletrodo modificado foi responsável pelo incremento do rendimento dos sensores amperométricos ${ }^{52,45}$.

Eletrodos modificados com filmes de CoHCF foram empregados para a determinação de glutationa in vivo ${ }^{41}$. Para coletar amostras e incrementar seletividade, os autores utilizaram sonda de microdiálise e separação cromatográfica, respectivamente. Este mesmo filme de Co foi empregado para modificar a superfície de microeletrodo de ouro para o estudo da oxidação catalítica de $\mathrm{NADH}^{53}$. O mecanismo proposto, baseado em clássico trabalho de Hin e Lowe ${ }^{54}$, pode ser representado como:

\section{$2 \mathrm{~K}\left\{\mathrm{CoFe}^{\mathrm{III}}(\mathrm{CN})_{6}\right\}+2 \mathrm{~K}^{+}+\mathrm{NADH} \rightarrow$ $2 \mathrm{~K}_{2}\left\{\mathrm{CoFe}^{\mathrm{II}}(\mathrm{CN})_{6}\right\}+\mathrm{NAD}^{+}+\mathrm{H}^{+}$}

onde NADH corresponde a forma reduzida da nicotinamidaadenina-dinucleotídeo ou simplesmente $\mathrm{NAD}^{+}$.

A oxidação eletrocatalítica da dopamina foi investigada em microdisco de platina $(\varnothing=30 \mu \mathrm{m})$ modificado com filme de $\mathrm{NiHCF}^{55}$. O processo eletroquímico envolvido pode ser apresentado como:

$$
\begin{gathered}
2 \mathrm{Na}\left\{\mathrm{Ni}^{\mathrm{II}}\left[\mathrm{Fe}^{\mathrm{III}}(\mathrm{CN})_{6}\right]\right\}+2 \mathrm{Na}^{+}+\text {dopamina }_{\text {reduzida }} \rightarrow \\
2 \mathrm{Na}_{2}\left\{\mathrm{Ni}^{\mathrm{II}}\left[\mathrm{Fe}^{\mathrm{II}}(\mathrm{CN})_{6}\right]\right\}+\text { dopamina }_{\text {oxidada }}
\end{gathered}
$$

Em presença de membrana polimérica à base de Nafion ${ }^{\mathrm{MR}}$ foi realizada a determinação de dopamina em presença de ácido ascórbico na razão de 50:1.

Glicose $^{56,57,58}$, etanol/metanol ${ }^{56,57}$ e glutamato ${ }^{59}$ foram determinados após modificação eletroquímica de eletrodo de carbono vítreo com filmes de $\mathrm{Fe}$ e $\mathrm{Fe} / \mathrm{Cu}-\mathrm{HCF}^{58}$. À superfície modificada foram depositadas as enzimas previamente imobilizadas em membranas à base de Nafion $^{\mathrm{MR}}$. O monitoramento de peróxido de hidrogênio produzido foi o parâmetro de medida para todas as aplicações. Ainda monitorando $\mathrm{H}_{2} \mathrm{O}_{2}$ via reação enzimática, filmes de $\mathrm{Ni}^{60}, \mathrm{Cr}^{61}$ e $\mathrm{Cu}^{62}$ modificando eletrodos de níquel, carbono vítreo e pasta da carbono respectivamente foram empregados para a determinação de glicose em sangue ${ }^{60}$ e fluídos biológi$\cos ^{62}$. O seguinte mecanismo pode ser apresentado para a determinação de peróxido de hidrogênio baseado na modificação do eletrodo de carbono vítreo modificado com filmes de $\mathrm{CrHCF}^{61}$ :

$$
\begin{gathered}
2 \mathrm{~K}_{2}\left\{\mathrm{Cr}^{\mathrm{II}}\left[\mathrm{Fe}^{\mathrm{II}}(\mathrm{CN})_{6}\right]\right\}+\mathrm{H}_{2} \mathrm{O}_{2}+2 \mathrm{H}^{+} \rightarrow \\
2 \mathrm{~K}\left\{\mathrm{Cr}^{\mathrm{III}}\left[\mathrm{Fe}^{\mathrm{II}}(\mathrm{CN})_{6}\right]\right\}+2 \mathrm{H}_{2} \mathrm{O}+2 \mathrm{~K}^{+}
\end{gathered}
$$

Etanol $^{54,63}$ e lactato ${ }^{64}$ (aplicação em fluídos biológicos) foram determinados empregando-se filmes de NiHCF modificando microeletrodo de ouro. Os métodos propostos foram baseados na oxidação eletrocatalítica de $\mathrm{NADH}^{54}$, espécie formada após reação enzimática (L-lactato $+\mathrm{NAD}^{+} \rightarrow \mathrm{NADH}+\mathrm{H}^{+}+$piruvato):

$$
\begin{gathered}
2 \mathrm{NaNiFe}^{\mathrm{III}}(\mathrm{CN})_{6}+2 \mathrm{Na}^{+}+\mathrm{NADH} \\
2 \mathrm{Na}_{2} \mathrm{NiFe}^{\mathrm{II}}(\mathrm{CN})_{6}+\mathrm{NAD}^{+}+\mathrm{H}^{+}
\end{gathered}
$$

Explorando a potencialidade dos eletrodos modificados com filmes de HCF para determinação de cátions não-eletroativos, 
Tabela 1. Aplicações amperométricas dos filmes baseados em metal-HCF.

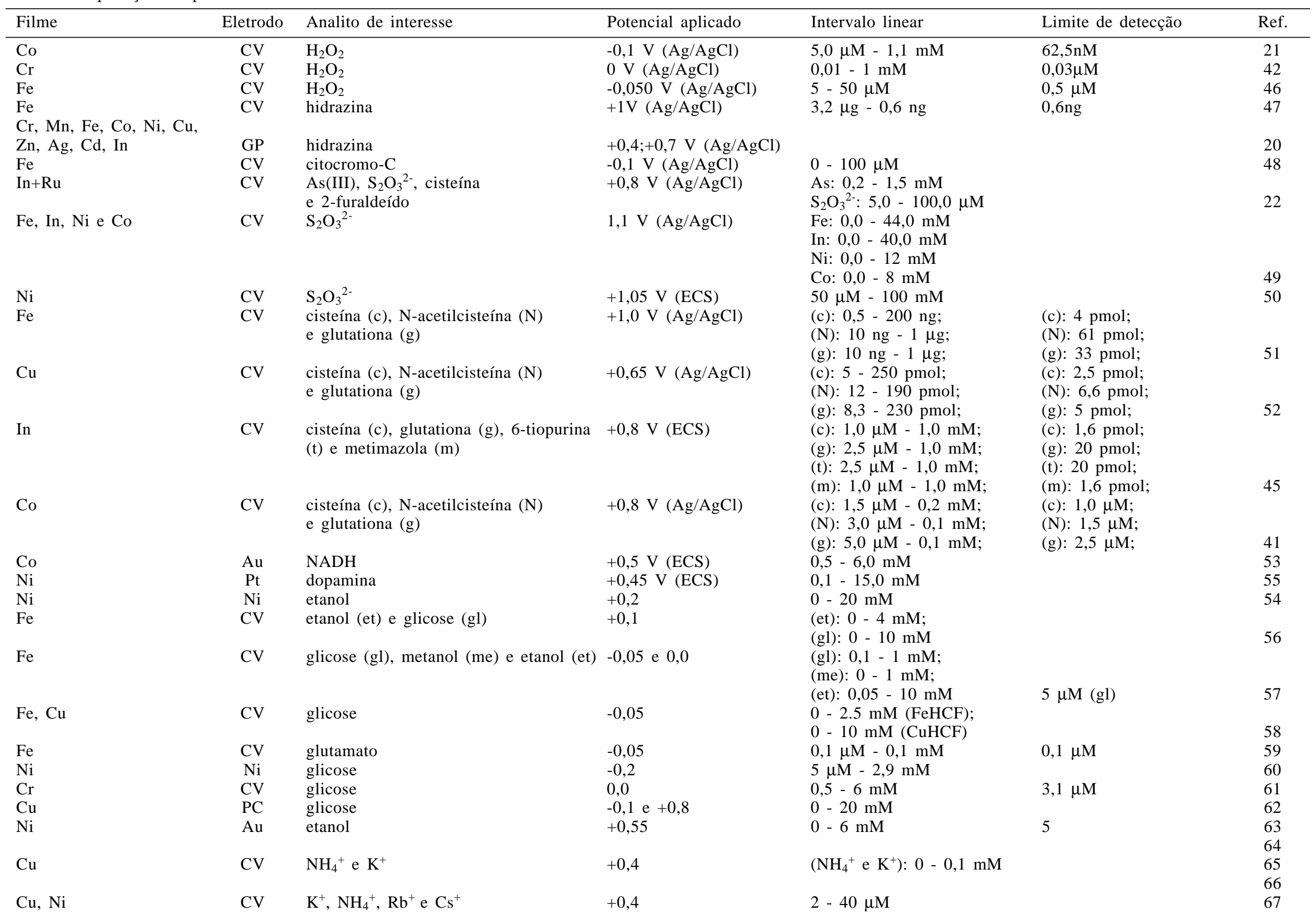


Tabela 2. Outras aplicações dos filmes baseados em metal-HCF.

\begin{tabular}{lllllc}
\hline Filme & Eletrodo & Espécie determinada & Detecção & Intervalo linear & Ref. \\
\hline $\mathrm{Cu}$ e $\mathrm{Ni}$ & $\mathrm{Cu}$ e $\mathrm{Ni}$ & $\mathrm{NH}_{4}^{+}$, metais alcalinos, terrosos e pesados & potenc. & & 68 \\
$\mathrm{Fe}$ & $\mathrm{In}$ & $\mathrm{Na}^{+}$ & potenc. & 69 \\
$\mathrm{Co}$ & $\mathrm{CV}$ & $\mathrm{K}^{+}$ & potenc. & $30 \mu \mathrm{M}-100 \mathrm{mM}$ & 70 \\
$\mathrm{Fe}$ & & ácido ascórbico & espectrof. & $0,1-10 \mathrm{mM}$ & 71 \\
\hline
\end{tabular}

potenc. - detecção potenciométrica; espectrof. - detecção espectrofotométrica;

Thomsen e Baldwin ${ }^{65}$ propuseram a detecção amperométrica de íons amônio e potássio em sistemas FIA e separação cromatográfica. O processo de oxidação/redução na superfície do eletrodo pode ser apresentado como:

$$
\mathrm{Cu}_{3}{ }_{3}\left[\mathrm{Fe}^{\mathrm{III}}(\mathrm{CN})_{6}\right]_{2}+2 \mathrm{e}^{-}+2 \mathrm{~K}^{+} \leftrightarrow \mathrm{K}_{2}\left\{\mathrm{Cu}_{3}{ }_{3}\left[\left(\mathrm{Fe}^{\mathrm{II}}(\mathrm{CN})_{6}\right)\right]_{2}\right.
$$

Trabalhando com eletrodo de Ni e carbono vítreo modificados, respectivamente, com filmes de $\mathrm{Ni}$ e $\mathrm{Cu}-\mathrm{HCF}$, estes autores apresentaram a determinação automatizada de $\mathrm{K}^{+}$e $\mathrm{NH}_{4}{ }^{+}$ (em urina), $\mathrm{K}^{+}$(soro sanguíneo). Ambos os eletrodos apresentaram resposta aos metais do grupo IA e íons amônio, mas somente o eletrodo modificado com CuHCF apresentou filme com estabilidade operacional para aplicações em fluxo ${ }^{66}$.

A determinação de traços de íons amônio em águas de chuva e lagoas foi conduzida usando filmes de $\mathrm{CuHCF}^{67}$. Para incrementar a seletividade e a sensibilidade, foi empregada uma câmara de difusão gasosa. Filmes de HCF em presença de $\mathrm{Cu}$ e $\mathrm{Ni}^{68}, \mathrm{Fe}^{69}$ e $\mathrm{Co}^{70}$ também foram utilizados para o desenvolvimento de eletrodos íon-seletivos, com as membranas sendo avaliadas em presença de metais alcalinos, alcalinos terrosos, metais pesados e íons amônio. Para a determinação potenciométrica de íons potássio, considerou-se que o potencial de pico (do par reversível, $\mathrm{K}_{2} \mathrm{Co}\left[\mathrm{Fe}(\mathrm{CN})_{6}\right]$ e $\mathrm{KCo}\left[\mathrm{Fe}(\mathrm{CN})_{6}\right.$ ) é função da atividade iônica (média) do íon potássio, e se o filme é tratado como uma solução sólida, a equação de Nernst pode ser apresentada como:

$$
\mathrm{E}=\mathrm{E}^{\circ}+\mathrm{RT} / \mathrm{nF} \ln \left[\mathrm{a}_{\mathrm{CoHCF}(\mathrm{ox})} \mathrm{a}_{\mathrm{K}+} / \mathrm{a}_{\mathrm{CoHCF}(\mathrm{red})}\right]
$$

Como última aplicação, cita-se a interessante determinação espectrofotométrica de ácido ascórbico em produtos farmacêuticos: filmes de PB foram quimicamente depositados em suportes não condutores (cubetas de plástico) com monitoramento do decréscimo da coloração devido à redução de $\mathrm{PB}$ a $\mathrm{PW}$ em presença de ascorbatos ${ }^{71}$. Este trabalho foi fruto de publicação anterior $^{72}$, onde os autores apresentaram as potencialidades das aplicações dos filmes de PB como sensores químicos ópticos.

\section{CONCLUSÃO}

Vários trabalhos têm explorado a caracterização dos filmes de metal-hexacianoferrato, incluindo estudos eletroquímicos e espectroscópicos, em presença de espécies químicas tanto orgânicas quanto inorgânicas. Conhecendo-se o comportamento eletroquímico do substrato nos mais diversos materiais de eletrodo modificados, e técnicas de eletrodeposição (para incrementar, entre outras, a estabilidade dos filmes) aumentam-se as expectativas para outras aplicações em química analítica. Neste sentido, os eletrodos modificados com filmes de HCF apresentam potencialidades para as mais diversas determinações sejam estas baseadas em medidas amperométricas, potenciométricas ou espectrofotométricas.

Apesar de os sistemas FIA serem uma importante ferramenta de trabalho para a rápida aquisição de dados, entre outras características, existe um número bastante limitado de trabalhos que fazem uso desta técnica. Acredita-se que é um campo também a ser explorado nas mais diversas aplicações, especialmente em presença das oxidases as quais quando devidamente imobilizadas nas superfícies dos eletrodos modificados constituem-se em (bio)-sensores extremamente adequados às determinações de amostras de alimentos, medicamentos, meio-ambiente, monitoramento industrial, entre outras.

Saliente-se que a estabilidade química, facilidade de preparação e baixo custo fazem os filmes de HCF atrativos para aplicações nas mais diversas áreas. Cita-se, por exemplo, que semi-condutores tais como sulfito de cádmio são estabilizados contra corrosão na presença de ferrocianetos, devido a formação de cianeto de cádmio, insolúvel. Na confecção de eletrodos íon-seletivos a não necessidade do uso de plastificantes, insolubilidade em solventes orgânicos, rápido tempo de resposta, possibilidade de miniaturização, etc. fazem dos eletrodos íon-seletivos baseados nestes filmes de HCF superiores aos de membrana líquida ou $\mathrm{PVC}^{70}$.

Os autores agradecem a European Commission (Project ERBIC15-CT98-0906) e ao Swedish Natural Science Research Council (NFR). ILM agradece ao Swedish Institute, Capes e Fapesp (Projeto 99/05621-4) pelo suporte financeiro. Os autores também agradecem a E.A.G. Zagatto e L. Kubota por valiosos comentários.

\section{REFERÊNCIAS}

1. American Cyanamid Company; The Chemistry of the Ferrocyanides; Beacon Press; New York, 1953.

2. Neff, V. D.; J. Electrochem. Soc. 1978, 125, 886.

3. Ellis, D.; Eckhoff, M.; Neff, V. D.; J. Phys. Chem. 1981, $85,1225$.

4. Itaya, K.; Uchida, I.; Neff, V. D.; Acc. Chem. Res. 1986, $19,162$.

5. Jiang, M.; Zhao, Z.; J. Electroanal. Chem. 1990, 292, 281.

6. Jin, Z.; Dong, S.; Electrochim. Acta 1990, 35, 1057.

7. Joseph, J.; Gomathi, H.; Rao, G. P.; Electrochim. Acta 1991, 36, 1537.

8. Sugimura, H.; Shimo, N.; Kitamura, N.; Masuhara, H.; Itaya, K.; J. Electroanal. Chem. 1993, 346, 147.

9. Gao, Z.; Bobacka, J.; Ivaska, A.; Electrochim. Acta 1993, $38,379$.

10. Moon, S. B.; Xidis, A.; Neff, V. D.; J. Phys. Chem. 1993, 97, 1634.

11. Kubota, L. T.; Gushikem, Y.; J. Electroanal. Chem. 1993, $362,219$.

12. Matsumoto, F. M.; Temperini, M. L. A.; Toma, H. E.; Electrochim. Acta 1994, 39, 385.

13. Dostal, A.; Schroder, U.; Scholz, F.; Inorg. Chem. 1995, 34, 1711.

14. Dostal, A.; Meyer, B.; Scholz, F.; Schroder, U.; Bond, A. M.; Marken, F.; Shaw, S. J.; J. Phys. Chem. 1995, 99, 2096.

15. Kulesza, P. J.; Doblhofer, K.; J. Electroanal. Chem. 1989, $274,95$.

16. Kuhnhardt, C.; J. Electroanal. Chem. 1994, 369, 71.

17. Ganzerli Valentini, M. T.; Meloni, S.; Maxia, V.; J. Inorg. Nucl. Chem. 1972, 34, 1427.

18. Loosneskovic, C.; Abousahl, S.; Fedoroff, M.; J. Mat. Sci. 1990, 25, 677.

19. Malik, M. A.; Kulesza, P. J.; Electroanal. 1996, 8, 113.

20. Narayanan, S. S.; Scholz, F.; Electroanal. 1999, 11, 465.

21. Lin, M. S.; Jan, B. I.; Electroanal. 1997, 9, 340.

22. Cataldi, T. R. I.; De Benedetto, G. E.; Bianchini, A.; Electroanal. 1998, 10,1163.

23. Dong, S.; Jin, Z.; J. Electroanal. Chem. 1988, 256, 193. 
24. Toma, H. E.; Lellis, F. T. P.; Spectroscopy Lett. 1988, $21,45$.

25. Cotton, F. A.; Wilkinson, G.; Advanced Inorganic chemistry; John Wiley \& Sons; New York, 1988.

26. Siperko, L. M.; Kuwana, T.; J. Electrochem. Soc. 1983, 130, 396.

27. Ogura, K.; Higasa, M.; Yano, J.; Endo, N.; J. Electroanal. Chem. 1994, 379, 373.

28. Ogura, K.; Endo, N.; Nakayama, M.; Ootsuka, H.; J. Electrochem. Soc. 1995, 142, 4026.

29. Scharf, U.; Grabner, E. W.; Electrochim. Acta 1996, 41, 233.

30. Itaya, K.; Shoji, N.; Uchida, I.; J. Am. Chem. Soc. 1984, 106, 3423.

31. Dong, S.; Chi, Q.; Anal. Chim. Acta 1995, 310, 429.

32. Karyakin, A. A.; Karyakina, E. E.; Gorton, L.; Electrochem. Comm. 1999, 1, 78.

33. Westbroek, P.; Temmerman, E.; Kiekens, P.; Anal. Chim. Acta 1999, 385, 423.

34. Schreck, S.; Dornenburg, H.; Knorr, D.; Food. Biotech. 1996, 10, 163.

35. Wang, J.; Lin, Y.; Chen, L.; Analyst 1993, 118, 277.

36. Gorton, L.; Csöregi, E.; Dominguez, E.; Emnéus, J.; Jönsson-Pettersson, G.; Marko-Varga, G.; Persson, P.; Anal. Chim. Acta 1991, 250, 203.

37. Calatayud, J. M.; Flow injection analysis of pharmaceuticals automation in the laboratory; Taylor \& Francis, Bristol, 1996.

38. Buser, H. J.; Schwarzenbach, D.; Petter, W.; Ludi, A.; Inorg. Chem. 1977, 16, 2704.

39. Boyer, A.; Kalcher, K.; Pietsch, R.; Electroanal. 1990, 2, 155 .

40. Garjonyte, R.; Malinauskas, A.; Sensors and Actuators 1999, B56, 93.

41. Shi, G.; Lu, J.; Xu, F.; Sun, W.; Jin, L.; Yamamoto, K.; Tao, S.; Jin, J.; Anal. Chim. Acta 1999, 391, 307.

42. Lin, M. S.; Tseng, T. F.; Shih, W. C.; Analyst 1998, 123,159

43. Bard, A. J.; Faulkner, L. R., Electrochemical Methods Fundamentals and Applications; John Wiley \& Sons, Inc., New York, 1980.

44. Wang, J.; Analytical Electrochemistry; VCH Publishers, Inc.; New York, 1994.

45. Zhang, S.; Sun, W.; Zhang, W.; Qi, W.; Jin, L.; Yamamoto, K.; Tao, S.; Jin, J.; Anal. Chim. Acta 1999, 386, 21.

46. Mattos, I. L.; Gorton, L.; Ruzgas, T.; Karyakin, A. A.; Anal. Sci. 2000, 16, 795.

47. Hou, W.; Wang, E.; Anal. Chim. Acta 1992, 257, 275.
48. Lu, W.; Wallace, G. G.; Karyakin, A. A.; Electroanal. 1998, 10, 472.

49. Chen, S.; J. Electroanal. Chem. 1996, 417, 145.

50. Zhou, X.; Wang, S.; Wang, Z.; Jiang, M.; Fresenius J. Anal. Chem. 1993, 345, 424.

51. Hou, W.; Wang, E.; J. Electroanal. Chem. 1991, 316, 155.

52. Zhou, J.; Wang, E.; Electroanal. 1994, 6, 29.

53. Cai, C.; Ju, H.; Chen, H.; J. Electroanal. Chem. 1995, 397, 185.

54. Yon Hin, B. F. Y.; Lowe, C. R.; Anal. Chem. 1987, 59, 2111.

55. Zhou, D.; Ju, H.; Chen, H.; J. Electroanal. Chem. 1996, $408,219$.

56. Karyakin, A. A.; Karyakina, E. E.; Gorton, L.; Bobrova, O. A.; Luckchova, L. V.; Gladilin, A. K.; Levashov, A. V.; Anal. Chem. 1996, 68, 4335.

57. Karyakin, A. A.; Karyakina, E. E.; Gorton, L.; Talanta 1996, 43, 1597.

58. Mattos, I. L.; Gorton, L.; Laurell, T.; Malinauskas, A.; Karyakin, A. A.; Talanta 2000, 52, 791.

59. Karyakin, A. A.; Karyakina, E. E.; Gorton, L.; Anal. Chem. (submitted).

60. Milardovic, S.; Kruhak, I.; Ivekovic, D.; Rumenjak, V.; Tkalcec, M.; Grabaric, B. S.; Anal. Chim. Acta 1997, 350, 91.

61. Lin, M. S.; Shih, W. C.; Anal. Chim. Acta 1999, 381, 183.

62. Wang, J.; Zhang, X.; Prakash, M.; Anal. Chim. Acta 1999, $395,11$.

63. Cai, C. X.; Xue, K. H.; Zhou, Y. M.; Yang, H.; Talanta 1997, 44, 339

64. Cai, C. X.; Ju, H.; Chen, H.; Anal. Lett. 1995, $28,809$. 65. Thomsen, K. N.; Baldwin, R. P.; Anal. Chem. 1989, 61, 2594. 66. Thomsen, K. N.; Baldwin, R. P.; Electroanal. 1990, 2, 263. 67. Liu, R.; Sun, B.; Liu, D.; Sun, A.; Talanta 1996, 43, 1049. 68. Tani, Y.; Eun, H.; Umezawa, Y.; Electrochim. Acta 1998, 43, 3431.

69. Aoki, K.; Miyamoto, T.; Ohsawa, Y.; Bull. Chem. Soc. Jpn 1989, 62, 1658.

70. Gao, Z.; Zhou, X.; Wang, G.; Li, P.; Zhao, Z.; Anal. Chim. Acta 1991, 244, 39.

71. Koncki, R.; Lenarczuk, T.; Glab, S.; Anal. Chim. Acta 1999, 379, 69.

72. Koncki, R.; Wolfbeis, O. S.; Sensors and Actuators 1998 , B51, 355 .

73. Mattos, I. L.; Lukachova, L. V.; Gorton, L.; Laurell, T.; Baust-Timpson, M.; Karyakin, A. A.; Talanta, no prelo. 